\title{
HSP-90 inhibitors promise to complement cancer therapies
}

By the end of the year, pivotal clinical trials will be well underway in a new class of drugs that inhibit heat shock protein 90 (HSP-90), a stress-induced molecule abundant in tumors and fungal infections. Two companies, Kosan Biosciences and NeuTec Pharma, are vying for first-in-field status with drugs that take different approaches to squelching HSP-90. Drugs addressing this class of drug target could prove a significant complement to existing cancer therapies but the first to market is likely to reap the most benefits.

Under key collaborative agreements with the US National Cancer Institute (NCI), Kosan, located in Hayward, California, is developing small-molecule anti-HSP-90 drugs targeted specifically against cancer. In contrast, NeuTec, located in Manchester, UK-acquired last June by Novartis of Basel-is developing monoclonal antibodies to fight fungal infections, with cancer as a secondary target. Phase 3 trials of NeuTec's recombinant human antibody Mycograb in fungal infections are recruiting subjects internationally; phase 2 trials of Mycograb combined with traditional chemotherapy in metastatic or recurrent breast cancer are also underway.

Meanwhile, a half-dozen other biotech companies have small molecule HSP-90 inhibitors in preclinical development (Table 1). "HSP-90 as a target is extraordinarily interesting and attractive because it's a factor in so many different tumor types," says Eric Schmidt, biotechnology analyst at Cowen and Company, in New York City, but "there hasn't been a whole lot of compelling data thus far."

HSP-90 first attracted attention as a potential cancer drug target in the early 1990s, when researchers discovered that it enables a host of proteins involved in cancer. "HSP-90 is a powerful and exciting mechanism," said Robert Johnson, Kosan's CEO. "It's important for normal cells and even more important for the survival of tumor cells." Formed in response to stress (and first seen in overheated cells), heat shock proteins protect other proteins_called clients-during their construction inside the cell. But HSP-90 also helps the same proteins survive when they go awry. "If you make a list of mutated molecules that can cause cancer, many are clients of HSP-90," says Len Neckers, a senior investigator at NCI.

In 1993, Neckers and his NCI colleagues discovered that a common, off-patent antibiotic, geldanamycin, could inhibit HSP-90's chaperoning function. The discovery launched an onslaught of research, and in 2001, NCI partnered with Kosan to develop geldanamycin

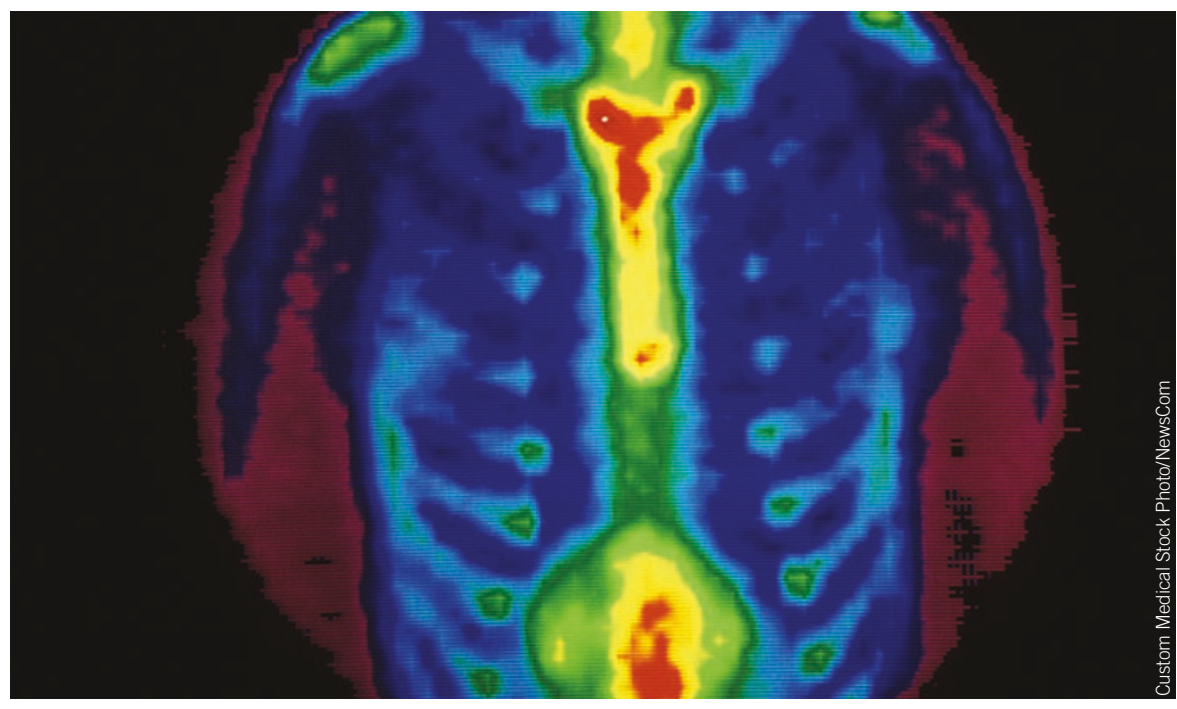

HSP-90 targeted drugs, about to be tested in multiple myeloma and breast cancer, could become complementary to traditional cancer therapies.

derivatives, a program that led to tanespimycin.

Kosan boasts that it has treated 600 patients with tanespimycin in five completed phase 1 trials and a handful of ongoing phase 2 trials that test the drug alone or in combination with chemotherapy or targeted agents. The trials cover a broad range of cancers, including advanced breast cancer, prostate cancer and an assortment of leukemias. Although none of the early results have been a "home run," notes Neckers, they have been promising enough to convince Kosan to invest in large-scale trials.

Kosan expects to launch a phase 3 trial in multiple myeloma patients of its first antiHSP-90 drug, tanespimycin, by the end of the year, and anticipates filing for US Food and Drug Administration (FDA) approval in 2009. The trial will test tanespimycin plus the standard myeloma therapy Velcade (bortezomib) against Velcade alone. Kosan is also developing a second generation anti-HSP-90 drug, alvespimycin, currently in early trials against metastatic breast cancer and other solid tumors. "One of the major challenges in treating multiple myeloma is the large number of patients with either primary or acquired resistance to
Velcade," says Asha Chanan-Khan of Roswell Park Cancer Institute, Buffalo, New York. "So we are encouraged by these early results."

The results point to an appealing aspect of HSP-90 inhibitors: their propensity to overcome drug resistance, comments Luke Whitesell, a pediatric oncologist at the University of Arizona, who was part of the Neckers' team that discovered geldanamycin's prowess for inhibiting HSP-90. For instance, leukemia cells that develop resistance to Gleevec (imatinib) "retain their sensitivity for geldanamycin and, if anything, become more sensitive to it."

Johnson is most enthusiastic about Kosan's second generation HSP-90 inhibitor, alvespimycin. "We believe it will help change treatment paradigms" in breast cancer, he told investors at the UBS Global Life Sciences conference in New York in September. Alvespimycin is "five to eight times more potent" than its first generation cousin, meaning patients can be given lower doses, and it can also be given orally (tanespimycin is intravenous). Johnson says that if all goes well, Kosan could file for FDA approval of alvespimycin in 2010.

Brian Vastag, Washington, DC
Table 1 Selected companies developing HSP90

\begin{tabular}{ll} 
Company & Drug (indication) \\
\hline Infinity Pharmaceuticals & IPI-504 \\
\hline Conforma Therapeutics & CNF-1010
\end{tabular}

Source: Adis R\&D Insight and company websites.
Stage of development

Phase I Multiple myeloma, gastrointestinal tumors

Phase I Chronic myeloid leukemia and solid tumors 